\section{Some Effects of Neutron Irradiation of Diamond}

Five type 2 diamonds, whose ability to transmit ultra-violet radiation and whose response as counters of $\alpha$-particles had already been examined, were irradiated by neutrons in B.E.P.O. at Harwell. Typical curves showing the variation in counting response (in terms of maximum pulse-height) with radiation dosage are shown in the graph. The general behaviour leaves no doubt that the counting response is progressively reduced with increasing neutron exposure. Similarly, photometric measurements showed that a uniform reduction occurred in the ultra-violet transmission.

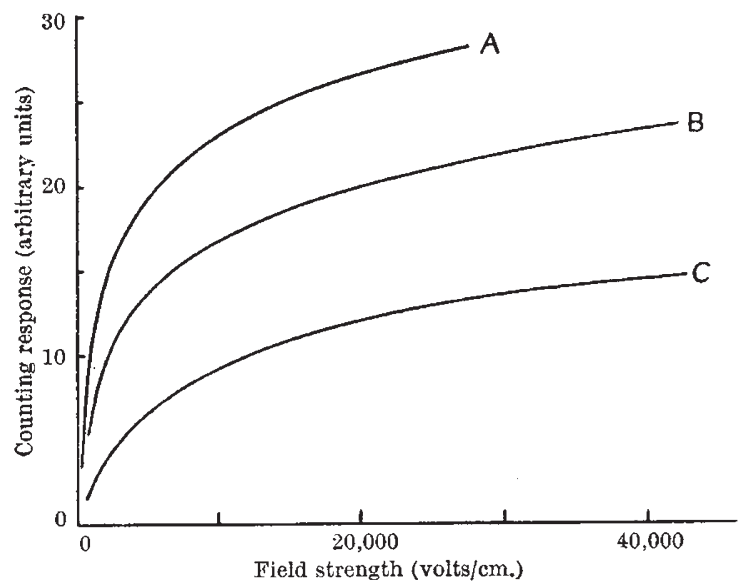

(A) Initially ; $(B)$ after $\frac{1}{2}$-hr. irradiation with neutrons: (C) after 2 -hr. irradiation with neutrons

The obvious interpretation is that additional traps are produced which reduce both the counting efficiency and the ultra-violet transmission. Some recovery in both these properties took place on heating the specimens ; but since heating resulted in no improvement in the counting properties of non-irradiated diamonds, it is clear that the intrinsic traps differ in some way from the traps produced by neutron irradiation.

A further feature was that neutron irradiation of the specimens resulted in a decrease in the overall insulation resistance, which was at least partially restored by heating.

These phenomena seem to be connected with those already reported by Griffiths, Owen and Ward ${ }^{1}$, and a fuller account of our experiments and their interpretation will be given elsewhere.

A. H. BenNy

F. C. Champion

Wheatstone Laboratory,

King's College, London. April 1.

1 Griffiths, J. H. E., Owen, J., and Ward, I. M., Nature, 173, 439 (1954).

\section{Electron Resonance in Ionospheric Waves}

Is an earlier communication ${ }^{1}$, a brief summary was given of some theoretical work pertaining to the travelling disturbances now regularly observed in the ionosphere ${ }^{2}$. A more detailed discussion has now been prepared for publication. and a new aspect of the investigation has been initiated. As this seems even more promising, and as it may well affect any further work in the reduction of observational data, its more important features are recorded here.

The earlier development was based on the assumption that the disturbances are basically hydrodynamic waves, and that the observed perturbations in the electron density are produced primarily by a resonant electric field generated magneto-hydrodynamically. In the new work, the hydrodynamic wave hypothesis is retained, but the observational selection is now attributed to a direct resonance in the electron oscillations, regardless of any electromagnetic fields which may be generated in the process. It is also assumed that any horizontal propagation must be relatively free from damping if the disturbance is to be observed and recorded by present methods.

The resonant oscillations most simply described are those which occur in an otherwise stationary atmosphere of uniform temperature, and they fall into two distinct classes. In the first, a velocity perturbation of the form $\exp i(\omega t-\mathbf{k} . \mathbf{r})$ is subject to the condition

$$
\omega=\gamma^{2} g^{2} C_{e}^{2} \sin ^{2} I / 2 C^{4} K_{e},
$$

where $\gamma$ is the specific heat ratio and $C$ the 'speed of sound' for the atmosphere, $K_{e}$ is the collisional frequency and $C_{e}$ the 'speed of sound' for electrons, $g$ is the acceleration due to gravity, and $I$ is the magnetic dip angle. On representing the horizontal component of $\mathbf{k}$ by $k_{h}$, its magnetic azimuth by $\theta$, and the vertically downwards component of $\mathbf{k}$ by $k_{v}$, it is further found that:

$$
\begin{aligned}
& k_{h}^{2} C^{2}\left[1+\cot ^{2} I \cos ^{2} \theta-(\gamma-1) g^{2} / \omega^{2} C^{2}\right]- \\
& k_{h} \gamma g \cot I \cos \theta+\gamma^{2} g^{2} / 2 C^{2}-\omega^{2}=0,
\end{aligned}
$$
and

$$
k_{v}=(1-i) \gamma g / 2 C^{2}-k_{h} \cot I \cos \theta .
$$

From the latter, it is apparent that hydrodynamic energy is uniformly distributed in height and that a downwards component of propagation is inherent. This component is reduced, however, and may even be reversed, if the poleward component of $\mathbf{k}$ becomes very large. The condition that the roots of (2) must be real will prevent such a reversal if $\omega$ is less than about $(\gamma-1)^{1 / 2} g \sin |I| / C$, and to a certain extent it also limits the possible range of $\omega$ or $\theta$.

Variations from the normal electron density $N_{0}$, which alone are relevant in the radio investigations, differ by a factor

$$
N_{0}\left[k_{v}+k_{h} \cot I \cos \theta+i \operatorname{grad}_{v} N_{0} / N_{0}\right]
$$

from the exponential form indicated above. Corrections for vertical variations in $N_{0}$ and $\operatorname{grad}_{v} N_{0}$ (the downwards component of grad $N_{0}$, negative in the regions accessible to direct probing) must therefore be included whenever $k_{v}$ is related to observation.

It is difficult to extract precise values of ionospheric parameters from the mean observational data published to date, but even approximate estimates reveal a considerable improvement over the earlier results. The large-scale disturbances detected in the $F_{2}$ region ${ }^{3}$ now lead to increased values for the scale height, $40 \mathrm{~km}$. or more, in closer agreement with other estimates; the earlier difficulty with low $\gamma$ 's appears to have been removed, and no subsidiary considerations need be introduced to explain the observed downwards progression. The collisional frequency can now be estimated if the electrons are taken to be at atmospheric temperature; the value thus obtained for the $F_{2}$-layer, several hundred per second, is in good agreement with other moasurements and theory 4 . 